\title{
Effects of $3 \%$ diquafosol sodium ophthalmic solution on higher-order aberrations in patients diagnosed with dry eye after cataract surgery
}

This article was published in the following Dove Press journal:

Clinical Ophthalmology

23 December 2016

Number of times this article has been viewed

\section{Yasushi Inoue Shintarou Ochi \\ Inoue Eye Clinic, Tamano, Okayama, Japan}

Correspondence: Yasushi Inoue Inoue Eye Clinic, I-|4-3I Uno,

Tamanoshi, Okayama, Japan

$\mathrm{Tel}+8|8633| 1030$

Fax +8189331 IIII4

Email inoue@oka.urban.ne.jp
Purpose: To evaluate the effects of diquafosol sodium ophthalmic solution 3\% (DQS) and artificial tears (AT) on higher-order aberrations (HOAs) in patients with dry eye after cataract surgery.

Design: This was a post hoc analysis of a previously conducted randomized clinical study.

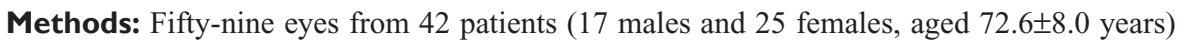
with verified or suspected dry eye at 4 weeks after cataract surgery were evaluated. The dry eye patients were randomly assigned to receive DQS or AT for 4 weeks. Tear breakup time (BUT), corneal and conjunctival fluorescein staining scores, and HOAs were analyzed before and after instillation. HOAs were measured consecutively for 10 seconds with a wavefront analyzer. Average HOAs, HOA fluctuations (fluctuation index [FI]) and changes in HOAs (stability index [SI]) were compared within and between the two groups.

Results: After 4 weeks of instillation, BUT significantly increased $(P=0.001)$ compared with preinstillation values in the DQS group, but not in the AT group. This increase in BUT in the DQS group was significantly greater than in the AT group $(P=0.014)$. Corneal and conjunctival fluorescein staining scores after instillation significantly improved compared with preinstillation values in the DQS group $(P=0.018)$. In HOAs, the cornea aberration changed from an upward curve (a sawtooth pattern) to an almost constant value (a stable pattern) in the DQS group, but not in the AT group. In FI and SI, there were no significant changes in either group; however, FI and SI were significantly lower in the DQS group than in the AT group (both, $P=0.004$ ).

Conclusion: The dry eye patients after cataract surgery had a visual dysfunction in HOAs. DQS is effective to treat dry eye disease after cataract surgery with improvement of visual function.

Keywords: cataract surgery, dry eye, tear film breakup time, higher-order aberrations, diquafosol ophthalmic solution

\section{Introduction}

After a long history of development and refining of the surgical procedure, today, cataract surgery is considered to be one of the safest and most effective ophthalmic surgical procedures, and it is performed on a large number of patients. However, many patients complain of subjective symptoms after cataract surgery, such as eye discomfort and foreign-body sensation. Effective treatment of patients with such symptoms is not established and it is a clinical problem that needs to be addressed promptly. ${ }^{1,2}$ Recent studies have revealed that these subjective symptoms are due to the surgery-induced dry eye. Dry eye is a chronic disease of tear fluid and the keratoconjunctival epithelium due to various causes. Tear film instability in dry eye is considered to cause subjective 
symptoms and results in impaired optical quality of the eye, such as higher-order aberrations (HOAs). ${ }^{3,4}$

For the treatment of dry eye, ophthalmic solutions are widely used. In 2010, 3\% diquafosol sodium ophthalmic solution (DQS) was approved for the treatment of dry eye in Japan. DQS promotes the secretion of water and mucin from conjunctival cells. Previous studies reported that DQS effectively treated various types of dry eye and improved the visual function of the dry eye patients. ${ }^{5,6}$

In dry eye after cataract surgery, as well, it was reported that the treatment with ophthalmic solutions including DQS was effective. ${ }^{7-9}$ Although effectiveness of DQS treatment on HOAs in dry eye has been reported, there is not enough evidence, especially in cataract surgery-induced dry eye.

In this study, to address the clinical problem regarding unexpected visual dysfunction induced by cataract surgery, we evaluated the effect of DQS on HOAs in postoperative dry eye patients compared with artificial tears (AT).

\section{Methods}

\section{Study design}

This study was a post hoc analysis of a previously conducted open-label randomized clinical study (The University Hospital Medical Information Network [UMIN] Clinical Trials Registry No UMIN000016060). This study was carried out in Inoue Eye Clinic in Okayama, Japan. This study was conducted in accordance with the Declaration of Helsinki. The study protocol was approved by the institutional review board of Tokyo Chidori Hospital in Tokyo, and all patients gave written informed consent. Information about this study was deposited in the UMIN Clinical Trials Registry (No UMIN000022332).

\section{Eligibility criteria}

The main inclusion criteria for the study participants were as follows: 1) age of $\geq 50$ years, 2) implemented age-related cataract surgery by phacoemulsification, 3) confirmed or suspected postoperative dry eye according to the 2006 Japanese Dry Eye Diagnostic Criteria, ${ }^{10}$ and 4) postoperative corneal and conjunctival fluorescein staining scores of $\geq 1$ (maximum score: 9 points). The main exclusion criteria were as follows: 1) history of laser-assisted in situ keratomileusis, blepharoplasty, or corneal transplantation, and 2) history of intraocular surgery or ocular injury within 1 year before the preoperative examination.

\section{Cataract surgery}

The operative field was preliminarily disinfected with a povidone-iodine solution at a concentration of $10 \%$ for skin and $0.625 \%$ for mucous membranes. Under topical anesthesia (administered as eye drops), a transconjunctival single-plane sclerocorneal or corneoscleral incision was made and an ophthalmic viscoelastic substance was subsequently injected. Then, an incision of the anterior capsule was subsequently made, phacoemulsification was conducted, and an intraocular lens was implanted. After the surgery; 0.1\% topical fluorometholone (Flumetholon ${ }^{\circledR}$; Santen Pharmaceutical Co., Ltd., Osaka, Japan) and 1.5\% levofloxacin eye drops (Cravit ${ }^{\circledR}$, Santen Pharmaceutical Co., Ltd.) were administered for 4 weeks, and $0.1 \%$ diclofenac sodium (Diclod ${ }^{\circledR}$ eye drops; Wakamoto Pharmaceutical Co., Ltd., Tokyo, Japan) was administered for 8 weeks.

\section{Examination of ocular surface and tear function}

Tear breakup time (BUT), corneal and conjunctival fluorescein staining scores, a subjective symptom questionnaire, and HOAs were examined before cataract surgery, 4 weeks after cataract surgery (immediately before DQS or AT instillation) and 8 weeks after cataract surgery (after 4 weeks of instillation). Schirmer test was carried out before and 4 weeks after surgery.

BUT measurement was performed using a wettable fluorescein strip. A BUT test measures the time (seconds) it takes for the tear film to disrupt and leave an exposed corneal surface. Three measurements for each eye were obtained using a slit-lamp microscope, and the mean value of the three measurements was calculated. Corneal and conjunctival fluorescein staining was evaluated in three regions (cornea as well as the temporal and nasal sides of conjunctiva) stained with fluorescein, and the staining in each region was assigned a score of 0-3 (maximum score: 9 points). Schirmer test was conducted on open eyes (with spontaneous blinking) without anesthesia.

Dry eye was diagnosed according to the Japanese diagnostic criteria. ${ }^{10}$ Verified dry eye was determined to have positive subjective symptoms, tear film abnormality (BUT $\leq 5$ seconds or Schirmer test $\leq 5 \mathrm{~mm}$ ), and positive fluorescein staining score ( $\geq$ score 3 ). Suspected dry eye was determined to have two of these three conditions.

\section{HOAs measurements}

Ocular and corneal HOAs were measured with a wavefront analyzer (KR-1W; Topcon Corp, Tokyo, Japan) automatically measuring and recording sequential wavefront aberrations. Serial measurement of total ocular HOAs was made every second for 10 seconds after a single eye blink. The observed HOAs for a 4-mm pupil were analyzed up to the 
sixth order by expanding the set of Zernike polynomials. Coma-like aberrations, spherical-like aberrations, and total HOAs were subsequently calculated. To assess the postblink ocular HOAs in dry eyes, the wave patterns of temporal changes in HOAs were classified into three types: a stable pattern (defined as maintaining an almost constant value with little variation), a fluctuating pattern (defined as having fluctuations in sequential values compared with a stable pattern without a tendency to increase or decrease), and a sawtooth pattern (defined as having total HOAs with an upward curve after blinking and decreasing at the blink). For the classification, a graph with the scale resolution of $0.01 \mu \mathrm{m}$ was plotted and a fluctuation was defined as the presence of zigzag configurations $\geq 0.02 \mu \mathrm{m}$ in width., ${ }^{3,11}$ The fluctuation index (FI: the average of the standard deviation of the ocular total HOAs), indicating the fluctuations in the total HOAs, and the stability index (SI: the slope of the linear regression line of the total ocular HOAs), quantifying the upward curve in the sawtooth pattern, were determined.

\section{Treatments for dry eyes}

Eligible patients received the dry eye treatment (DQS or AT) for 4 weeks from the visit 4 weeks after cataract surgery. They were randomly assigned to one of two treatment groups using a permuted block method. The DQS group received DQS eye drops (Diquas ${ }^{\circledR}$; Santen Pharmaceutical Co., Ltd.) six times daily and the AT group received AT eye drops (Mytear ${ }^{\circledR}$; Senju Pharmaceutical Co., Ltd.) six times daily. AT Mytear ophthalmic solution contains sodium chloride $5.5 \mathrm{mg}$, potassium chloride $1.6 \mathrm{mg}$, dried sodium carbonate $0.6 \mathrm{mg}$, dibasic sodium phosphate hydrate $1.8 \mathrm{mg}$, and boric acid $12 \mathrm{mg}$ in $1 \mathrm{~mL}$.

\section{Statistical analysis}

Baseline variables were summarized as number (\%) for categorical variables, and as mean \pm standard deviation, or as median (interquartile range) for continuous variables based on assumed distribution. These variables were compared between groups using Fisher's exact test, the Student's $t$-test, or the Mann-Whitney $U$ test as appropriate. As BUT, corneal and conjunctival fluorescein staining scores, FI, and SI were time series variables, a repeated measures analysis using estimated marginal means and $95 \%$ confidence interval in a generalized linear mixed model with log link function was used in the analysis. In the model, the study subjects were considered a random effect and time, treatment types, and the interaction term of time and treatment types were considered fixed effects. Multiplicity was corrected using the Bonferroni method.
A $P$-value $<0.05$ was considered statistically significant. All of the statistical analyses were conducted using IBM SPSS Statistics 22.0 software (International Business Machines Corp, Armonk, NY, USA).

\section{Results Study participants}

A total of 42 patients were enrolled in this study, diagnosed with dry eye 4 weeks after cataract surgery. They had undergone cataract surgery between 3 February and 2 October 2015. All of them fulfilled the eligibility criteria. HOAs in 26 eyes from 20 patients in the DQS group and 33 eyes from 22 patients in the AT group were evaluated. The diagnosis of dry eye was confirmed by fulfilling all of the three criteria in 13 eyes (50\%) in the DQS group and in 20 eyes $(60.6 \%)$ in the AT group. The diagnosis was suspected by fulfilling two of the three criteria in 13 eyes (50\%) in the DQS group and in 13 eyes (39.4\%) in the AT group. Table 1 presents the characteristics of study patients and the data of study eyes. Patients' characteristics were generally well-balanced between the two groups, with the exception of presurgery staining score $(P=0.047)$. All of the patients adhered to the study protocol and completed the treatment. No adverse events were observed.

\section{Changes in dry eye-associated parameters}

Table 2 presents the temporal changes in the estimated marginal mean of BUT, the corneal and conjunctival fluorescein staining scores, and the Schirmer test scores for the DQS and AT groups. At 4 weeks after cataract surgery (immediately before DQS or AT instillation), BUT was significantly decreased when compared with preoperative BUT in both the DQS and AT groups ( $P=0.019$ and $P<0.001$, respectively). At 4 weeks of DQS instillation, compared with before instillation, BUT was significantly increased $(P=0.001)$, whereas BUT after AT instillation did not change significantly from

Table I Baseline patient characteristics and disposition of the study eyes

\begin{tabular}{llll}
\hline & $\begin{array}{l}\text { DQS group } \\
(\mathbf{n = 2 0 )}\end{array}$ & $\begin{array}{l}\text { AT group } \\
(\mathbf{n}=\mathbf{2 2})\end{array}$ & P-value \\
\hline Male, n (\%) & $10(50.0)$ & $7(31.8)$ & $0.346^{\mathrm{a}}$ \\
Female, n (\%) & $10(50.0)$ & $15(68.2)$ & \\
Age (years), mean \pm SD & $74.9 \pm 8.1$ & $70.6 \pm 7.6$ & $0.087^{\mathrm{b}}$ \\
$\begin{array}{l}\text { Duration (minutes) of } \\
\text { surgery, median (IQR) }\end{array}$ & $7.00(6.00,8.00)$ & $7.00(6.00,7.25)$ & $0.407^{\mathrm{c}}$ \\
$\begin{array}{l}\text { Left eyes, n (\%) } \\
\text { Right eyes, n (\%) }\end{array}$ & $12(46.2)$ & $18(54.5)$ & $0.604^{\mathrm{a}}$ \\
\hline
\end{tabular}

Notes: 'Fisher's exact test. 'Student's t-test. 'Mann-Whitney's U-test.

Abbreviations: AT, artificial tears; DQS, diquafosol sodium ophthalmic solution; $I Q R$, interquartile range; SD, standard deviation. 
Table 2 BUT, corneal and conjunctival fluorescein staining score, and Schirmer test score

\begin{tabular}{|c|c|c|c|}
\hline & $\begin{array}{l}\text { Before cataract } \\
\text { surgery (I) }\end{array}$ & $\begin{array}{l}\text { Four weeks after } \\
\text { surgery (2) }\end{array}$ & $\begin{array}{l}\text { After DQS/AT } \\
\text { instillation (3) }\end{array}$ \\
\hline \multicolumn{4}{|l|}{ BUT (seconds) } \\
\hline \multicolumn{4}{|l|}{ DQS } \\
\hline EM mean $(95 \% \mathrm{Cl})$ & $2.9(2.27-3.65)$ & $2.12(1.62-2.72)$ & $3.3(2.6-4.12)$ \\
\hline$P$-value (I vs $2 ; \mid$ vs $3 ; 2$ vs 3 ) & $0.019 * ; 0.312 ; 0.001 * *$ & & \\
\hline \multicolumn{4}{|l|}{ AT } \\
\hline EM mean $(95 \% \mathrm{Cl})$ & $2.97(2.38-3.67)$ & $1.92(1.48-2.43)$ & $2.18(1.7-2.74)$ \\
\hline$P$-value (I vs $2 ; I$ vs $3 ; 2$ vs 3 ) & $<0.00$ I**; $0.008^{* * ;} ; 0.314$ & & \\
\hline \multicolumn{4}{|l|}{ DQS vs AT } \\
\hline$P$-value & 0.877 & 0.582 & $0.014 *$ \\
\hline$P$-value for interaction & $0.035^{*}$ & & \\
\hline \multicolumn{4}{|l|}{ Corneal and conjunctival staining } \\
\hline \multicolumn{4}{|l|}{ score } \\
\hline \multicolumn{4}{|l|}{ DQS } \\
\hline EM mean $(95 \% \mathrm{Cl})$ & $1.04(0.59-1.49)$ & $2.39(1.94-2.84)$ & $1.77(1.32-2.22)$ \\
\hline$P$-value (I vs $2 ;$ I vs $3 ; 2$ vs 3 ) & $<0.00 I^{* *} ; 0.005^{* *} ; 0.018^{*}$ & & \\
\hline \multicolumn{4}{|l|}{ AT } \\
\hline EM mean $(95 \% \mathrm{Cl})$ & $1.66(1.25-2.07)$ & $2.42(2.01-2.83)$ & $1.99(I .58-2.4 I)$ \\
\hline$P$-value (I vs $2 ; \mid$ vs $3 ; 2$ vs 3$)$ & $0.001^{* *} ; 0.145 ; 0.064$ & & \\
\hline \multicolumn{4}{|l|}{$\mathrm{DQS}$ vs $\mathrm{AT}$} \\
\hline$P$-value & $0.047^{*}$ & 0.922 & 0.475 \\
\hline$P$-value for interaction & 0.219 & & \\
\hline \multicolumn{4}{|l|}{ Schirmer test score } \\
\hline \multicolumn{4}{|l|}{ DQS } \\
\hline EM mean $(95 \% \mathrm{Cl})$ & $13.55(10.29-17.83)$ & II.7| (8.90-|5.4I) & - \\
\hline$P$-value (I vs 2 ) & 0.260 & & \\
\hline \multicolumn{4}{|l|}{ AT } \\
\hline EM mean $(95 \% \mathrm{Cl})$ & $10.63(8.24-13.71)$ & $10.21(7.92-13.17)$ & - \\
\hline$P$-value (I vs 2 ) & 0.723 & & \\
\hline \multicolumn{4}{|l|}{ DQS vs AT } \\
\hline$P$-value & 0.212 & 0.474 & \\
\hline$P$-value for interaction & 0.536 & & \\
\hline
\end{tabular}

Notes: DQS or AT was instilled from the visit 4 weeks after cataract surgery for 4 weeks. Schirmer test was carried out before and 4 weeks after surgery. Mixed model post hoc test with Bonferroni correction: $* P<0.05$; $* * P<0.0$ I; $* * * P<0.001$.

Abbreviations: AT, artificial tears; BUT, tear breakup time; $\mathrm{Cl}$, confidence interval; DQS, diquafosol sodium ophthalmic solution; EM mean, estimated marginal mean.

before instillation. After instillation, BUT was significantly greater in the DQS group than in the AT group $(P=0.014)$.

The corneal and conjunctival fluorescein staining score before DQS or AT instillation was significantly increased compared with preoperative score in both the DQS and AT groups $(P<0.001$ and $P=0.001$, respectively). At 4 weeks of DQS instillation, compared with before instillation, the staining score was significantly decreased $(P=0.018)$, whereas the staining score after AT instillation did not change significantly from before instillation.

Schirmer test scores did not change significantly 4 weeks after cataract surgery in both the DQS and AT groups. There were no significant differences in Schirmer test scores between the DQS and AT groups.

\section{Changes in HOAs, $\mathrm{FI}$, and $\mathrm{SI}$}

Figure 1 presents the temporal changes in HOAs. Four weeks after the surgery in both groups, the wave patterns in total, coma-like, and spherical-like HOAs changed into an upward curve, so that the wave pattern transitioned from a fluctuating pattern to a sawtooth pattern. At 4 weeks of DQS instillation, the wave pattern calmed down into a stable pattern. In contrast, the upward curve in the sawtooth pattern swelled after AT instillation.

Table 3 presents the temporal changes in the estimated marginal mean of FI and SI. At 4 weeks after cataract surgery, FI did not change significantly from preoperative values in both groups. At 4 weeks of instillation, compared with before instillation, FI showed a tendency to decrease in the DQS group, whereas FI showed a tendency to increase in the AT group. However, neither of these changes was statistically significant. After instillation, FI was significantly lower in the DQS group than in the AT group $(P=0.004)$.

At 4 weeks after cataract surgery, SI did not significantly change from preoperative values in both groups. After 4 weeks of DQS instillation, compared with before 


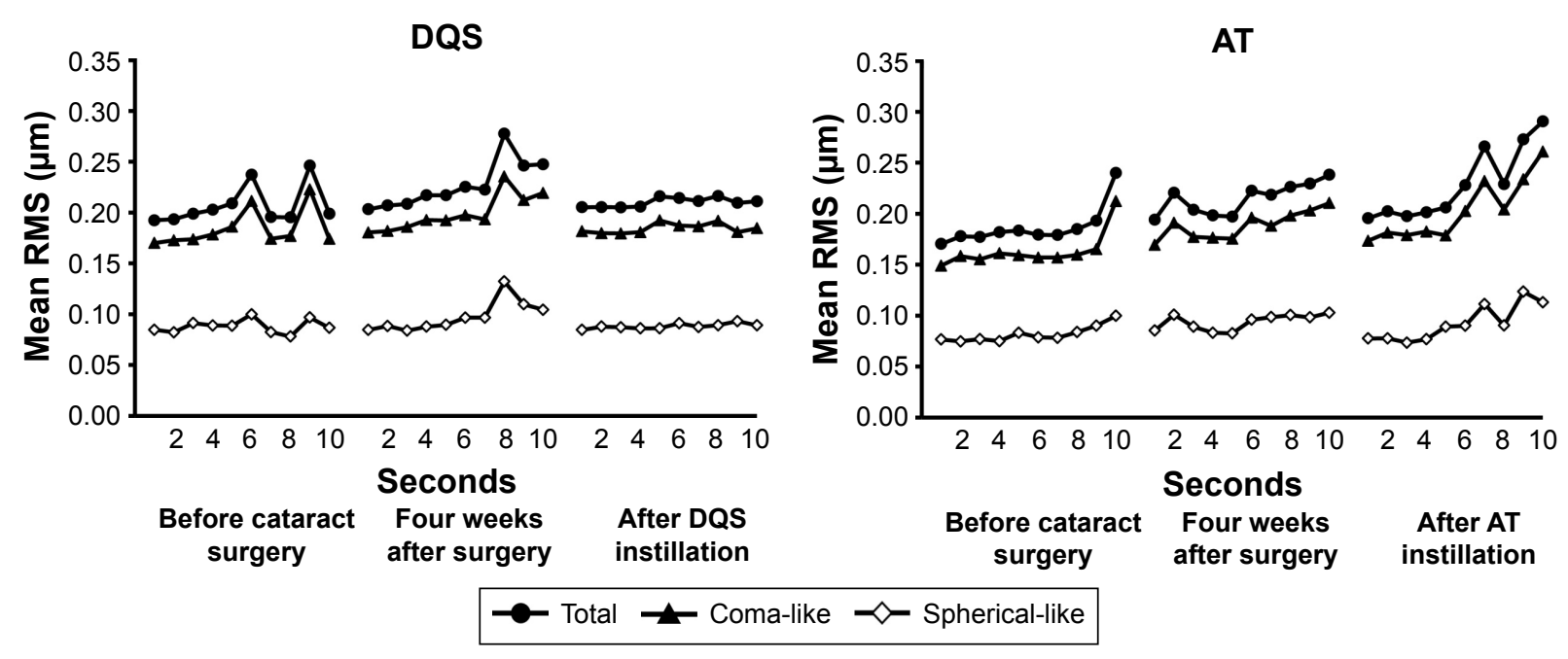

Figure I Temporal changes in higher-order aberrations.

Note: DQS or AT was instilled from the visit 4 weeks after cataract surgery for 4 weeks.

Abbreviations: AT, artificial tears; DQS, diquafosol sodium ophthalmic solution; RMS, root mean square.

instillation, SI did not significantly change, whereas SI after AT instillation significantly increased from before instillation. After instillation, SI was significantly lower in the DQS group than in the AT group $(P=0.004)$.

\section{Discussion}

Cataract surgery often adversely affects the tear film status manifested as decreased BUT and increased corneal and conjunctival staining scores, subsequently leading to the diagnosis of postoperative dry eye. ${ }^{1,12-15}$ In patients with postoperative dry eye in this study, as well, both BUT and corneal and conjunctival staining scores were significantly deteriorated compared with preoperative levels after cataract surgery. Phacoemulsification can modulate or block neurogenic responses on the ocular surface, thereby decreasing tear secretion. ${ }^{16,17}$ The impaired tear film status observed in our study was presumably due to protracted destabilizing effects of cataract surgery on the tear film.

Previous reports have demonstrated the close association of dry eye with HOAs. Destabilized tear film in dry

Table 3 Fluctuation index and stability index

\begin{tabular}{|c|c|c|c|}
\hline & $\begin{array}{l}\text { Before cataract } \\
\text { surgery (I) }\end{array}$ & $\begin{array}{l}\text { Four weeks after } \\
\text { surgery (2) }\end{array}$ & $\begin{array}{l}\text { After DQS/AT } \\
\text { instillation (3) }\end{array}$ \\
\hline \multicolumn{4}{|l|}{ Total fluctuation index } \\
\hline \multicolumn{4}{|l|}{ DQS } \\
\hline EM mean $(95 \% \mathrm{Cl})$ & $0.025(0.016-0.040)$ & $0.029(0.018-0.047)$ & $0.017(0.010-0.023)$ \\
\hline$P$-value (I vs $2 ; I$ vs $3 ; 2$ vs 3 ) & $0.637 ; 0.186 ; 0.087$ & & \\
\hline \multicolumn{4}{|l|}{ AT } \\
\hline EM mean $(95 \% \mathrm{Cl})$ & $0.032(0.021-0.049)$ & $0.029(0.019-0.045)$ & $0.049(0.032-0.075)$ \\
\hline$P$-value (I vs $2 ;$ I vs $3 ; 2$ vs 3 ) & $0.717 ; 0.132 ; 0.071$ & & \\
\hline \multicolumn{4}{|l|}{ DQS vs AT } \\
\hline$P$-value & 0.438 & 0.976 & $0.004 * *$ \\
\hline$P$-value for interaction & $0.020 *$ & & \\
\hline \multicolumn{4}{|l|}{ Total stability index } \\
\hline \multicolumn{4}{|l|}{ DQS } \\
\hline EM mean $(95 \% \mathrm{Cl})$ & $0.002(-0.002-0.007)$ & $0.007(0.002-0.012)$ & $0.001(-0.004-0.005)$ \\
\hline$P$-value (I vs $2 ;$ I vs $3 ; 2$ vs 3 ) & $0.163 ; 0.588 ; 0.054$ & & \\
\hline \multicolumn{4}{|l|}{ AT } \\
\hline EM mean $(95 \% \mathrm{Cl})$ & $0.004(0.000-0.008)$ & $0.004(0.000-0.008)$ & $0.01(0.006-0.014)$ \\
\hline$P$-value (I vs $2 ; I$ vs $3 ; 2$ vs 3 ) & $0.958 ; 0.046 * ; 0.04 I^{*}$ & & \\
\hline \multicolumn{4}{|l|}{ DQS vs AT } \\
\hline$P$-value & 0.598 & 0.332 & $0.004 * *$ \\
\hline$P$-value for interaction & $0.016 *$ & & \\
\hline
\end{tabular}

Notes: DQS or AT was instilled from the visit 4 weeks after cataract surgery for 4 weeks. Mixed model post hoc test with Bonferroni correction: $* P<0.05$; $* * P<0.0$ I. Abbreviations: AT, artificial tears; Cl, confidence interval; DQS, diquafosol sodium ophthalmic solution; EM mean, estimated marginal mean. 
eye increases HOAs, thereby impairing visual function. ${ }^{18,19}$ Ocular HOAs were significantly more prevalent in dry eyes than in normal eyes. ${ }^{20}$ In dry eye characterized by decreased BUT, the sawtooth pattern was observed in which total, coma-like, and spherical-like HOAs significantly increased over time compared with normal eyes. ${ }^{11,21}$ In this study, an upward curve was observed to shape a sawtooth pattern in temporal change in HOAs after cataract surgery, indicating impaired optical quality in postoperative dry eye as observed in various types of dry eye reported.

These findings suggest that stabilizing tear film while preserving tear film homogeneity is crucial to treat dry eye and to maintain visual function. DQS exerts its effects on the conjunctival epithelium and goblet cells and promotes the secretion of both water and mucin, thereby stabilizing the tear film..$^{22,23}$ In postoperative dry eye patients in our study, DQS instillation significantly improved BUT and corneal and conjunctival staining scores, stabilizing all the coma-like, spherical-like, and total HOAs to a stable pattern. In contrast, AT instillation did not significantly improve BUT or staining scores; moreover, the sawtooth pattern observed in postoperative HOAs became more prominent after AT instillation. These results demonstrate well the effectiveness of 4-week treatment with DQS to stabilize tear film and improve optical quality of the eyes in patients with dry eye following cataract surgery. This study was conducted under the usual surgery settings. The drugs used after cataract surgery (eg, diclofenac) might have affected symptoms. Further studies are needed to determine long-term effects of DQS in this population.

\section{Acknowledgment}

This study was financially supported by Santen Pharmaceutical Co., Ltd., Osaka, Japan.

\section{Disclosure}

YI has received consulting fees and honoraria for lectures from Pfizer, Kaneka Medix, NIDEK, MACHIDA Endoscope, Senju, Nitto Medic, and Kowa. Inoue Eye Clinic, wherein $\mathrm{YI}$ is the director and SO is an employee, has received grants for research in ocular diseases from multiple pharmaceutical companies (Santen, Otsuka, Alcon, and AMO). The authors report no other conflicts of interest in this work.

\section{References}

1. Hardten DR. Dry eye disease in patients after cataract surgery. Cornea. 2008;27(7):855.

2. Li XM, Hu L, Hu J, Wang W. Investigation of dry eye disease and analysis of the pathogenic factors in patients after cataract surgery. Cornea. 2007;26(Suppl 1):S16-S20.
3. Koh S, Maeda N, Hirohara Y, et al. Serial measurements of higher-order aberrations after blinking in patients with dry eye. Invest Ophthalmol Vis Sci. 2008;49(1):133-138.

4. Kaido M, Matsumoto Y, Shigeno Y, Ishida R, Dogru M, Tsubota K. Corneal fluorescein staining correlates with visual function in dry eye patients. Invest Ophthalmol Vis Sci. 2011;52(13):9516-9522.

5. Koh S, Maeda N, Ikeda C, et al. Effect of diquafosol ophthalmic solution on the optical quality of the eyes in patients with aqueous-deficient dry eye. Acta Ophthalmol. 2014;92(8):e671-e675.

6. Kaido M, Uchino M, Kojima T, Dogru M, Tsubota K. Effects of diquafosol tetrasodium administration on visual function in short break-up time dry eye. J Ocul Pharmacol Ther. 2013;29(6):595-603.

7. Baek J, Doh SH, Chung SK. The Effect of Topical Diquafosol Tetrasodium 3\% on Dry Eye After Cataract Surgery. Curr Eye Res. 2016;41(10): $1281-1285$.

8. Lee JH, Song IS, Kim KL, Yoon SY. Effectiveness and Optical Quality of Topical $3.0 \%$ Diquafosol versus $0.05 \%$ Cyclosporine A in Dry Eye Patients following Cataract Surgery. J Ophthalmol. 2016; 2016:8150757.

9. Park DH, Chung JK, Seo du R, Lee SJ. Clinical Effects and Safety of 3\% Diquafosol Ophthalmic Solution for Patients With Dry Eye After Cataract Surgery: A Randomized Controlled Trial. Am J Ophthalmol. 2016;163:122-131.

10. Shimazaki J, Tsubota K, Kinoshita S, Ohashi Y. [Definition and diagnosis of dry eye 2006.] Atarashii Ganka. 2007;24:181-184. Japanese.

11. Koh S, Maeda N, Hirohara Y, et al. Serial measurements of higherorder aberrations after blinking in normal subjects. Invest Ophthalmol Vis Sci. 2006;47(8):3318-3324.

12. Oh T, Jung Y, Chang D, Kim J, Kim H. Changes in the tear film and ocular surface after cataract surgery. Jpn J Ophthalmol. 2012;56(2): $113-118$.

13. Luchs JI, Nelinson DS, Macy JI; LAC-07-01 Study Group. Efficacy of hydroxypropyl cellulose ophthalmic inserts (LACRISERT) in subsets of patients with dry eye syndrome: findings from a patient registry. Cornea. 2010;29(12):1417-1427.

14. Cho YK, Kim MS. Dry eye after cataract surgery and associated intraoperative risk factors. Korean J Ophthalmol. 2009;23(2):65-73.

15. Vital MC, Belloso M, Prager TC, Lanier JD. Classifying the severity of corneal ulcers by using the "1, 2, 3" rule. Cornea. 2007;26(1):16-20.

16. Khanal S, Tomlinson A, Esakowitz L, et al. Changes in corneal sensitivity and tear physiology after phacoemulsification. Ophthalmic Physiol Opt. 2008;28(2):127-134.

17. Sitompul R, Sancoyo GS, Hutauruk JA, Gondhowiardjo TD. Sensitivity change in cornea and tear layer due to incision difference on cataract surgery with either manual small-incision cataract surgery or phacoemulsification. Cornea. 2008;27(Suppl 1):S13-S18.

18. Rieger G. The importance of the precorneal tear film for the quality of optical imaging. Br J Ophthalmol. 1992;76(3):157-158.

19. Koh S, Maeda N, Ninomiya S, et al. Paradoxical increase of visual impairment with punctal occlusion in a patient with mild dry eye. J Cataract Refract Surg. 2006;32(4):689-691.

20. Montes-Mico R, Caliz A, Alio JL. Wavefront analysis of higher order aberrations in dry eye patients. J Refrac Surg. 2004;20(3):243-247.

21. Koh S, Maeda N, Hori Y, et al. Effects of suppression of blinking on quality of vision in borderline cases of evaporative dry eye. Cornea. 2008;27(3):275-278.

22. Fujihara T, Murakami T, Nagano T, Nakamura M, Nakata K. INS365 suppresses loss of corneal epithelial integrity by secretion of mucin-like glycoprotein in a rabbit short-term dry eye model. J Ocul Pharmacol Ther. 2002;18(4):363-370.

23. Fujihara T, Murakami T, Fujita H, Nakamura M, Nakata K. Improvement of corneal barrier function by the P2Y(2) agonist INS365 in a rat dry eye model. Invest Ophthalmol Vis Sci. 2001;42(1):96-100. 
Clinical Ophthalmology

\section{Publish your work in this journal}

Clinical Ophthalmology is an international, peer-reviewed journal covering all subspecialties within ophthalmology. Key topics include: Optometry; Visual science; Pharmacology and drug therapy in eye diseases; Basic Sciences; Primary and Secondary eye care; Patien Safety and Quality of Care Improvements. This journal is indexed on

PubMed Central and CAS, and is the official journal of The Society of Clinical Ophthalmology (SCO). The manuscript management system is completely online and includes a very quick and fair peer-review system, which is all easy to use. Visit http://www.dovepress.com/ testimonials.php to read real quotes from published authors. 